\title{
CONSIDÉRATIONS PARASITOLOGIQUES
}

\section{SUR LA CÉNUROSE CÉRÉBRALE,}

\section{A PROpos D'UN NOUVEAU CAS}

\author{
Par J. RANQUE et R.-N. NICOLI
}

Depuis l'observation princeps de Pierre Marie, Foix et E. Brumpt (1913), la cénurose cérébrale humaine n'a été signalée que très rarement. Des recherches bibliographiques nous ont permis d'en retrouver huit cas que nous étudierons plus loin.

Cependant, Becker et Jacobson (1951) pensent que cette affection est « relativement commune » dans les régions d'élevage du mouton. Ils ont eu l'occasion d'en opérer successivement trois cas certains en Afrique du Sud. De plus, chez onze autres malades, la symptomatologie leur a fait soupçonner l'existence d'une cénurose cérébrale, sans que le diagnostic pût en être vérifié par examen parasitologique.

Si l'on admet cette façon de voir, il y aurait donc des rapports épidémiologiques entre la cénurose cérébrale et le kyste hydatique, en tant que maladies humaines.

Rappelons que le Tænia multiceps Leske, 1780 vit à l'état adulte dans la seconde moitié de l'intestin grêle du chien et des carnivores voisins. Sa larve est un cénure : cystique polycéphale, dont les scolex sont groupés en amas plus ou moins considérables, dans un kyste rempli de liquide. Son hôte normal est représenté par les ovins qui l'hébergent dans leur encéphale, où il provoque généralement une maladie bien connue en pathologie vétérinaire sous le nom de tournis. On trouve ce cénure occasionnellement chez d'autres ruminants, exceptionnellement chez divers mammifères, notamment l'homme.

Remarquons en passant que, dans cette dernière éventualité, l'homme ne saurait constituer un hôte intermédiaire efficient, tant pour des raisons épidémiologiques faciles à comprendre que par suite de la malformation fréquente des scolex dans le cénure cérébral humain.

Ann. de Parasitologie, T. $\mathrm{XXX}, \mathrm{N}^{\circ} 1-2 .-1955$. 
Chez l'hôte normal, il n'existe généralement qu'un seul cénure, tandis que, chez les hòtes anormaux, on peut en trouver une quantité plus ou moins considérable.

Du fait des anomalies rencontrées dans les cénures humains, une identification exclusivement morphologique peut présenter de grosses difficultés, d'où nécessité d'avoir recours à des caractères biologiques (localisation des parasites) et épidémiologiques, pour préciser le diagnostic.

Voici l'observation du cas de cénurose cérébrale que nous avons identifié, et qui représente le neuvième connu en pathologie humaine. L'étude clinique en a été faite par le $D^{r}$ Duplay, qui a opéré le malade, et l'examen histo-pathologique par Mme VitalBérard, qui a bien voulu nous confier l'identification du parasite et remettre la pièce aux collections de notre laboratoire. Nous les en remercions vivement. Nous résumons brièvement leur étude (1) :

Par. Ch., 42 ans, cultivateur et berger dans les Alpes-Maritimes. Adressé au $\mathrm{D}^{r}$ Duplay, neuro-chirurgien à Nice, pour céphalée et troubles psychiques à type de confusion mentale, évoluant progressivement depuis plusieurs mois.

Au moment de l'hospitalisation, incapacité totale de travail, apathie considérable, avec désorientation temporo-spatiale et incontinence des sphincters.

A relever, dans les antécédents, un traumatisme crânien en 1952. Examen nerveux strictement négatif, mais œdème papillaire net à droite, très discret à gauche. A l'électroencéphalogramme, tracé profondément altéré, traduisant une atteinte basilaire prédominant à gauche.

Examen hématologique pratiquement normal, sauf légère hyperleucocytose à 10.000 .

Artériographie cérébrale gauche n'apportant aucun élément particulier.

Ventriculographie : énorme hydrocéphalie bilatérale, non tendue, symétrique; le troisième ventricule n'est jamais injecté.

Une encéphalographie fractionnée montre le blocage de l'air au niveau de la base. Absence d'injection de la citerne otochiasmatique.

Le 20 novembre 1953, intervention sous perfusion neuroplégique. Scalp et volet frontal gauche. La dure-mère est tendue. Ponction du ventricule gauche. Ouverture de la dure-mère et descente sur la région chiasmatique engainée dans un magma adhérentiel, avec nombreuses vésicules hyalines de dimensions variables. Dégagement progressif de la lame susoptique, qui est ouverte. Prélèvement et fixation en vue d'examen. Fermeture.

(1) Duplay (J.), Bérard-Badie (M.) et Ranque (J.). - Soc. Oto-Neuto-Ophtalmologie, Nice, 31 octobre 1954. A paraître dans Revue d'O.N.O. 
L'intervention ne modifie en rien le cours de la maladie. Décès le 29 novembre 1953.

A l'autopsie, la convexité est normale, les ventricules sont dilatés ; mais la face antérieure du troisième ventricule et du tronc cérébral est engluée par un agglomérat de vésicules opalescentes, du même type que celles trouvées à l'intervention.

Ces formations d'aspect parasitaire sont incluses dans la leptoméninge. La méninge est le siège d'un processus inflammatoire à lymphocytes extrêmement important. Il existe même de petits îlots de macrophages (sidérophages et lipophages). Absence d'éléments éosinophiles.

Le parenchyme nerveux sous-jacent ne présente pas d'anomalies, en dehors d'une hyperplasie de l'astroglie protoplasmique de la couche moléculaire. La leptoméninge et le cortex sous-jacent à l'envahissement parasitaire sont très congestifs.

\section{Examen parasitologique}

Les pièces nous ont été communiquées après fixation dans le formol salé, ce qui a rendu toute expérimentation impossible.

$1^{\circ}$ Etude du fragment de biopsie. - Il s'agit d'une tumeur irrégulièrement sphérique, bosselée, ayant environ $5 \mathrm{~mm}$. de diamètre. Elle présente des prolongements (fig. 1), fréquemment observés dans les cénures d'hôtes anormaux. Ce fragment se compose d'une cuticule tapissée par un tissu parenchymateux, condensé par endroits, plus lâche en d'autres. Elle était pleine de liquide avant fixation. Elle contient trois scolex (fig. 2), émergeant d'une masse parenchymateuse et irrégulièrement développée.

On y voit des crochets fragmentés, mal ordonnés, de taille et de forme très variables, au nombre de 24 environ (fig. $3,4,5$ ).

$2^{\circ}$ Etude de la pièce prélevée a l'Autopsie. - Toute la région basilaire est enveloppée par un magma blanchâtre, contenant de très nombreuses vésicules de taille relativement petite $(5$ à $10 \mathrm{~mm}$. de diamètre), irrégulièrement sphériques, dont les dimensions ont été peut-être réduites par la fixation (fig. 6 à 10). Exceptionnellement, l'une mesurait $30 \mathrm{~mm}$. (fig. 9, 10).

Une série de coupes vertico-frontales nous permet de préciser la topographie de la tumeur parasitaire et son envahissement à partir de la région basilaire.

Sur la coupe passant par la région pré-chiasmatique (fig. 11), on note la présence de dix vésicules s'engageant dans les vallées sylviennes gauche et droite. 


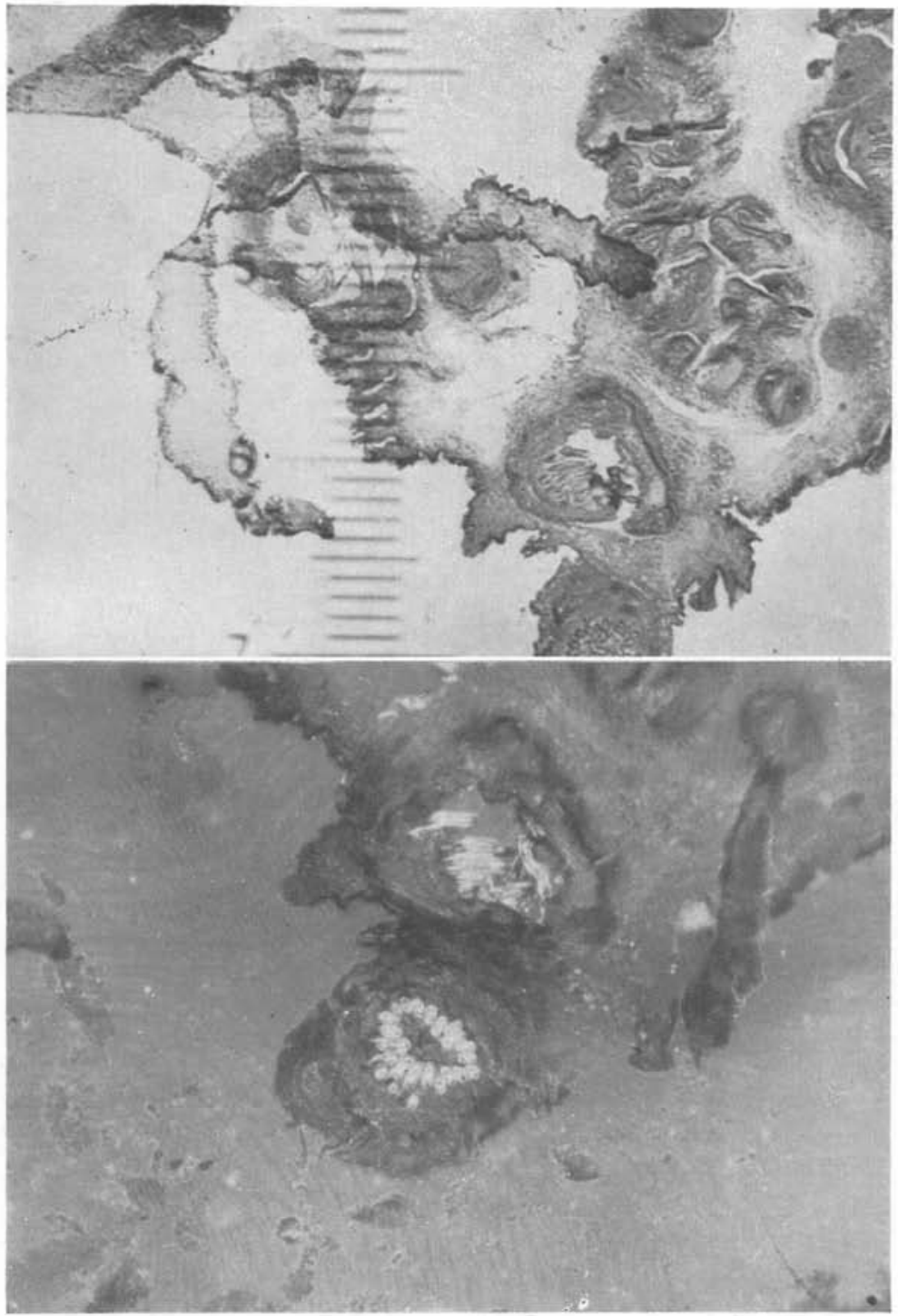

Fig. 1 (En haut). - Coupe de la biopsie à faible grossissement.

Fic. 2 (En bas). - Coupe de biopsie. Photo sur fond noir. 

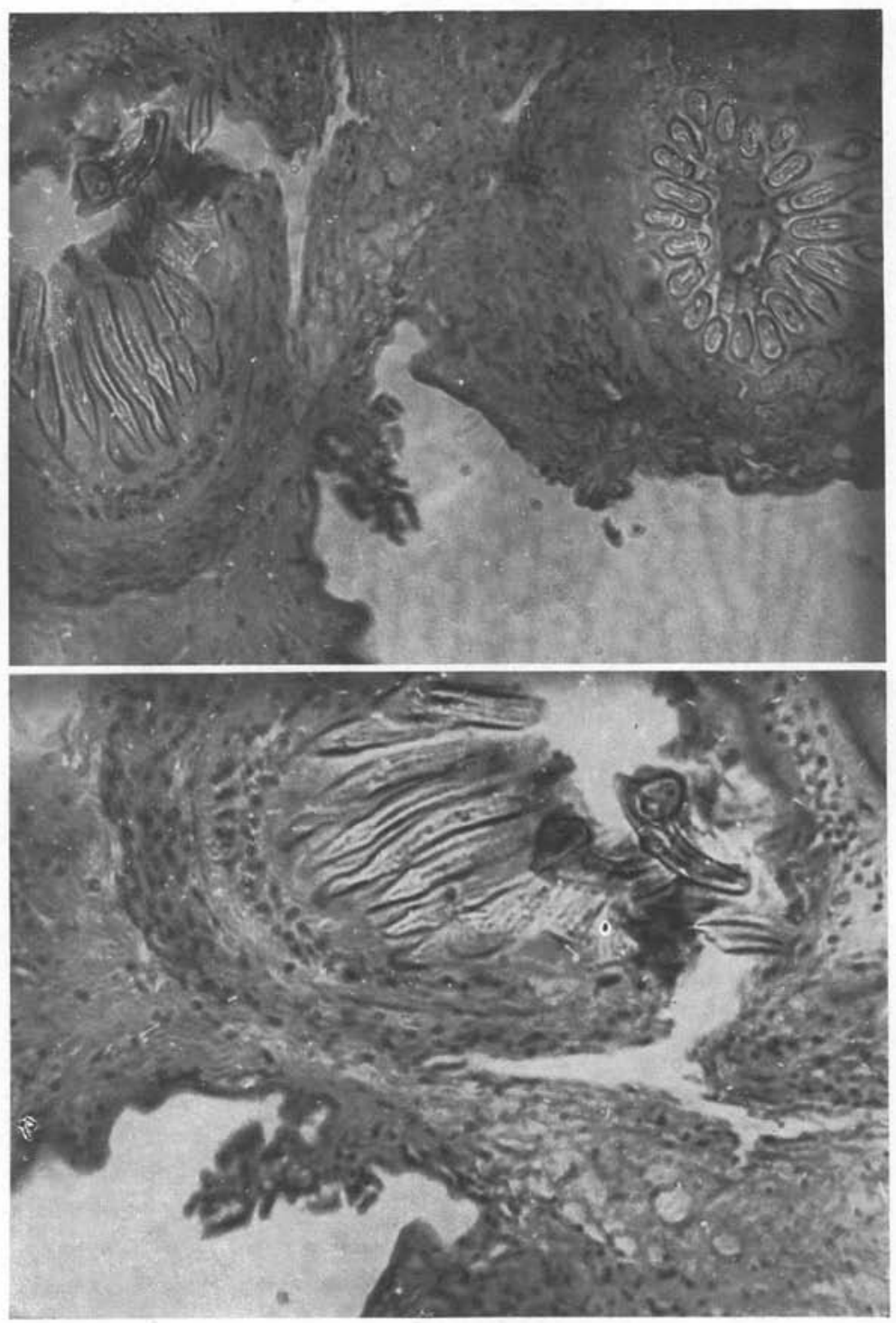

FIG. 3. - Coupe de biopsie. Grossissement moyen.

Fig. 4. - Coupes de biopsie. Fort grossissement. 


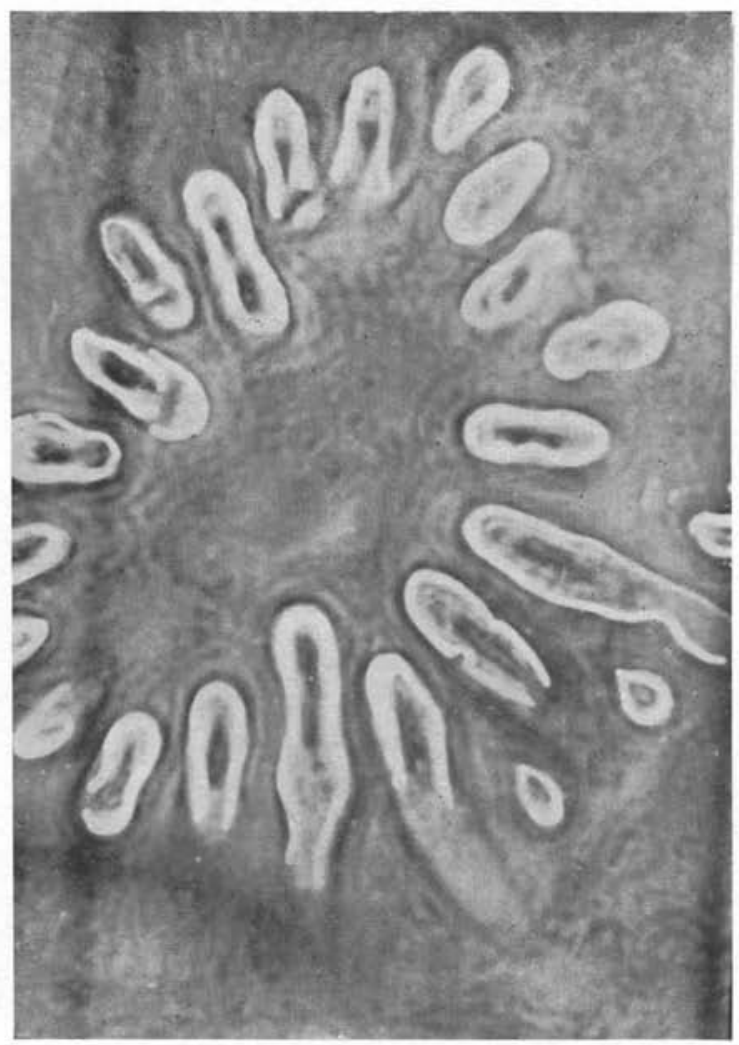

FIG. 5. - Coupes de biopsie. Fort grossissement.

Il en est de même sur une coupe passant par la commissure antérieure et le plein développement du noyau caudé (fig. 12), où il existe une infiltration parasitaire de la leptoméninge. Sur la face interne du lobe temporal gauche, on trouve aussi une formation kystique de la grosseur d'un petit pois, ayant refoulé le cortex sousjacent. Les deux vallées sylviennes sont complètement envahies par des amas opalescents intra-méningés de nature parasitaire (fig. 13).

Sur les coupes passant par le pulvinar (fig. 14), le sillon de l'hippocampe est envahi par les kystes parasitaires.

Le tronc cérébral est entouré par une gangue épaisse avec des vésicules très apparentes.

$\mathrm{Ni}$ atteinte parenchymateuse, ni prolongement postérieur entre le cervelet et les hémisphères cérébraux. 


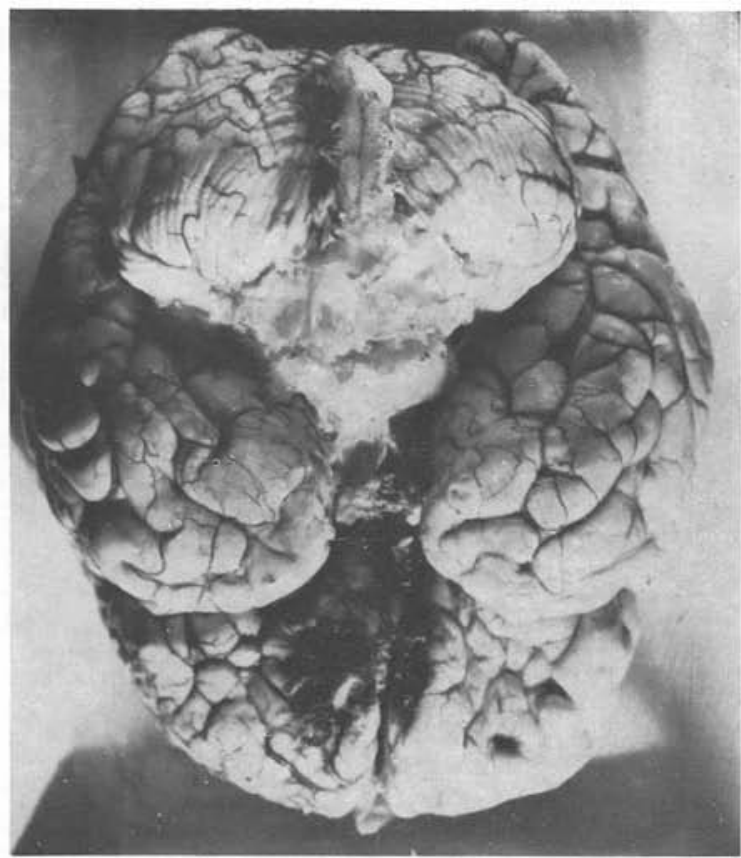

FIG. 6. - Face inférieure du cerveau.

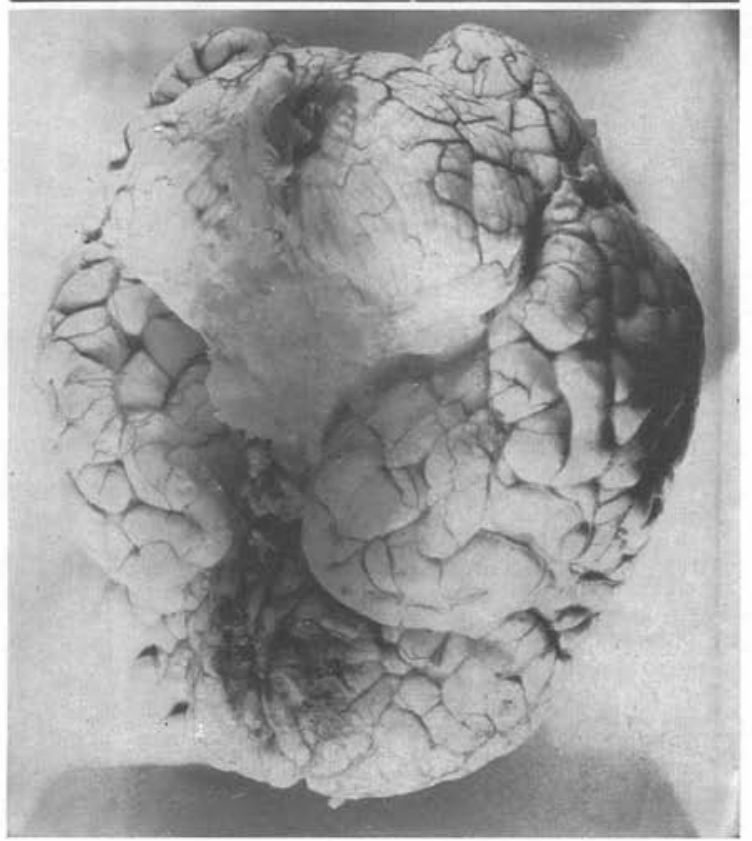

FIG. 7. - Cerveau, vu de trois-quarts. 


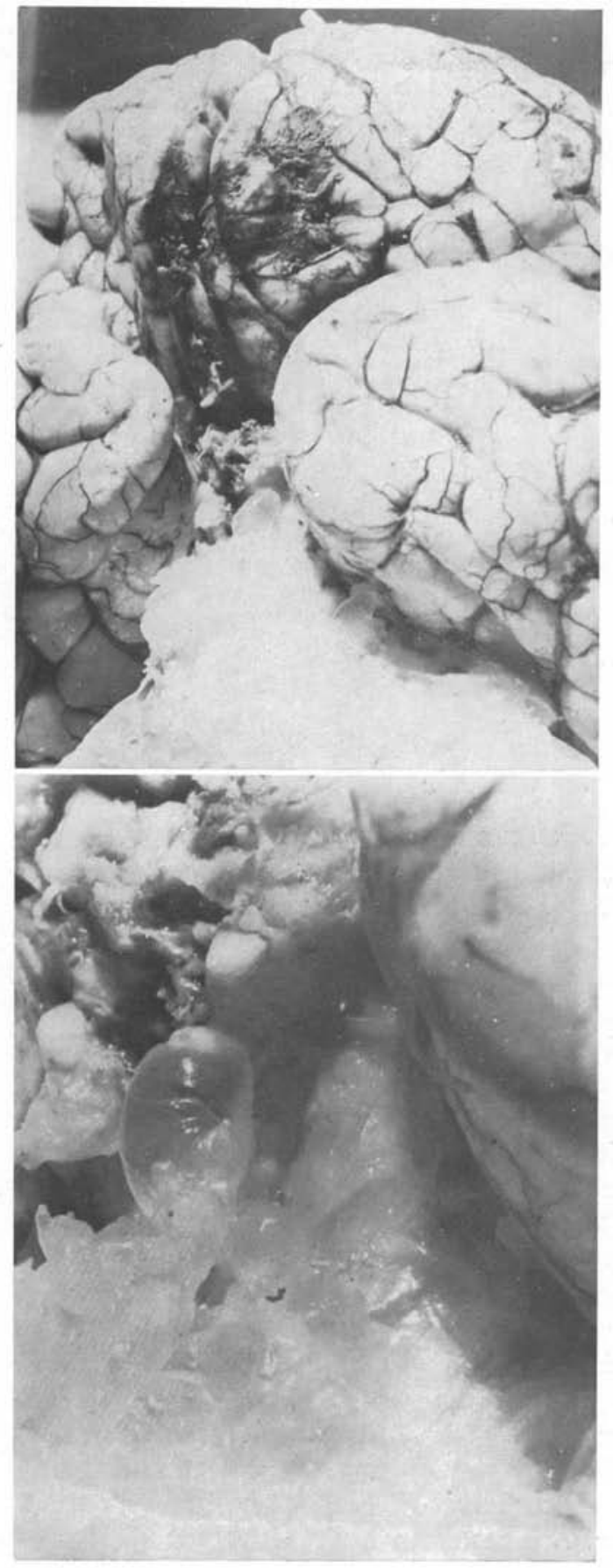

Fig. 8. - Région basilaire.

Fig. 9. - Cénure dans la région chiasmatique. 


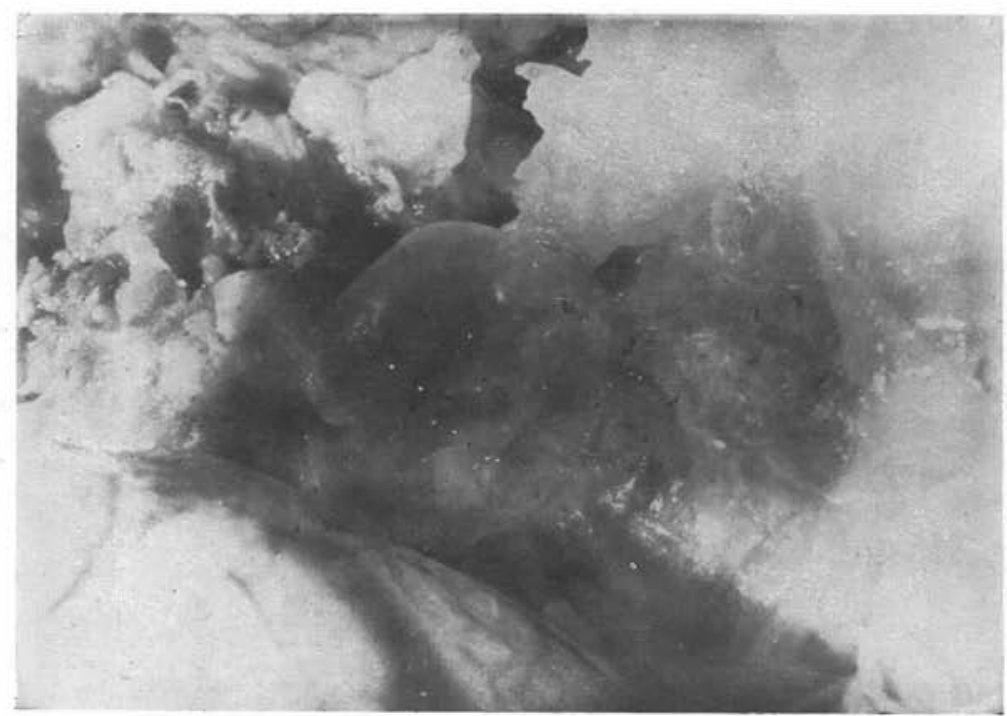

FIG. 10. - Cénure à l'entrée de la scissure de Sylvius.

Les vésicules dégénérées sans crochets sont rencontrées dans la région du pont de Varole, tandis que nous avons pu mettre en évidence des vésicules fertiles, dont une d'aspect très normal, dans le fond des vallées sylviennes. Nous pensons que lorqu'on étudie un cénure chez un hôte occasionnel, il est indispensable d'examiner le plus grand nombre possible de scolex, si l'on n'a pas la chance de trouver dès le début des crochets caractéristiques. C'est d'ailleurs une règle générale pour toute recherche helminthologique.

Il est curieux de constater que la biopsie préliminaire, au niveau de la région chiasmatique, ait permis de recueillir une vésicule à trois scolex, alors qu'ultérieurement, à l'examen systématique nécropsique, pratiqué en ce lieu, on n'a pu trouver que des éléments stériles.

Les vésicules normales sont irrégulièrement sphériques. Leur diamètre approximatif varie de 5 à $15 \mathrm{~mm}$. Elles émettent des prolongements moins marqués que dans les régions antérieure et superficielle.

La dilacération de ces vésicules nous a permis de mettre en liberté des scolex au nombre de 4 à 5 par vésicule (fig. 15, 16, 17).

Ces scolex mesurent en moyenne $980 \mu$. Leurs ventouses ont $150 \mu$ comme diamètre moyen. Le rostre porte une double rangée de cro- 


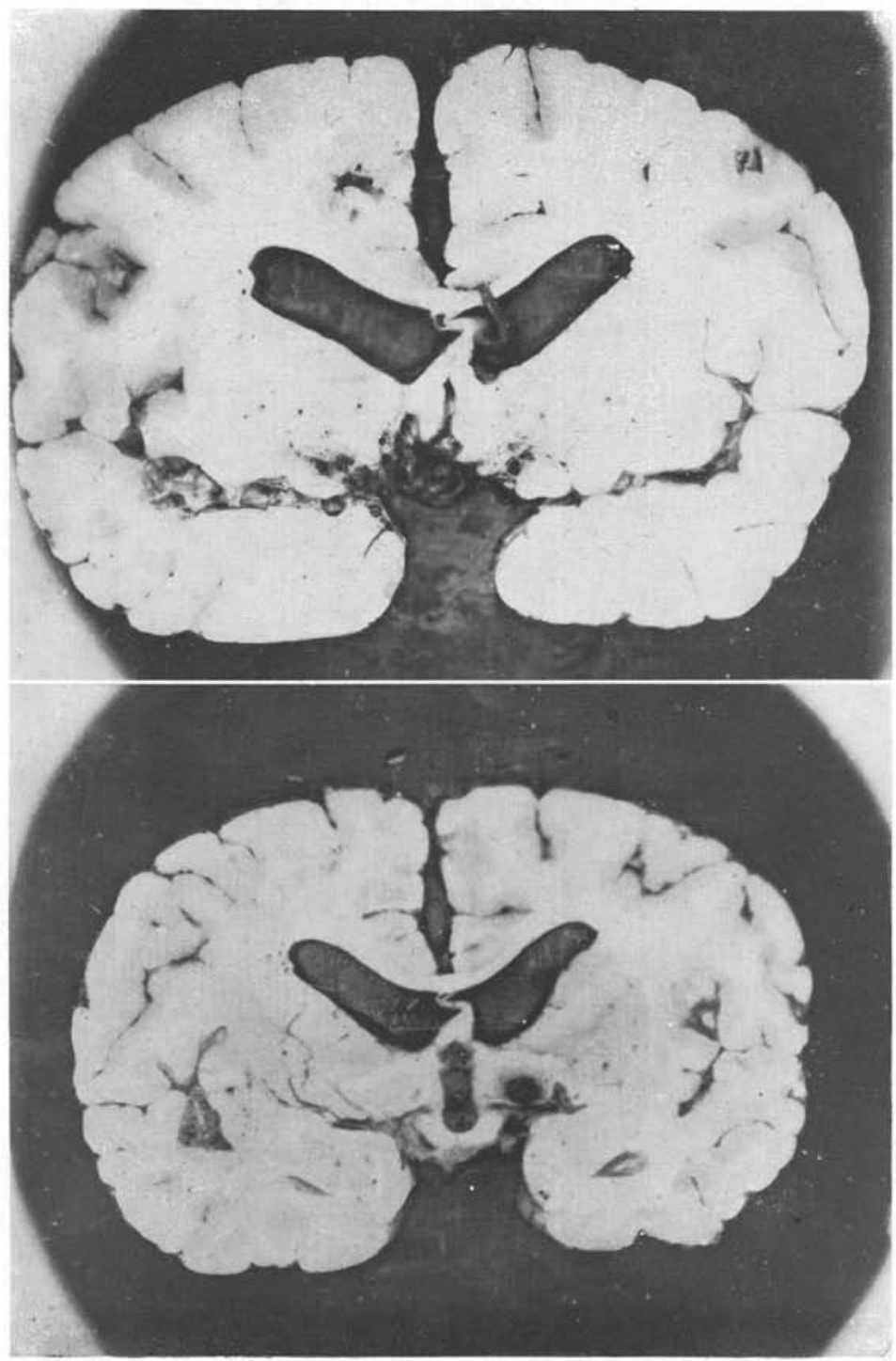

Fıg. 11 (En haut). - Coupe vertico-frontale de la région préchiasmatique. Fig. 12 (En bas). - Coupe passant par la commissure antérieure. 

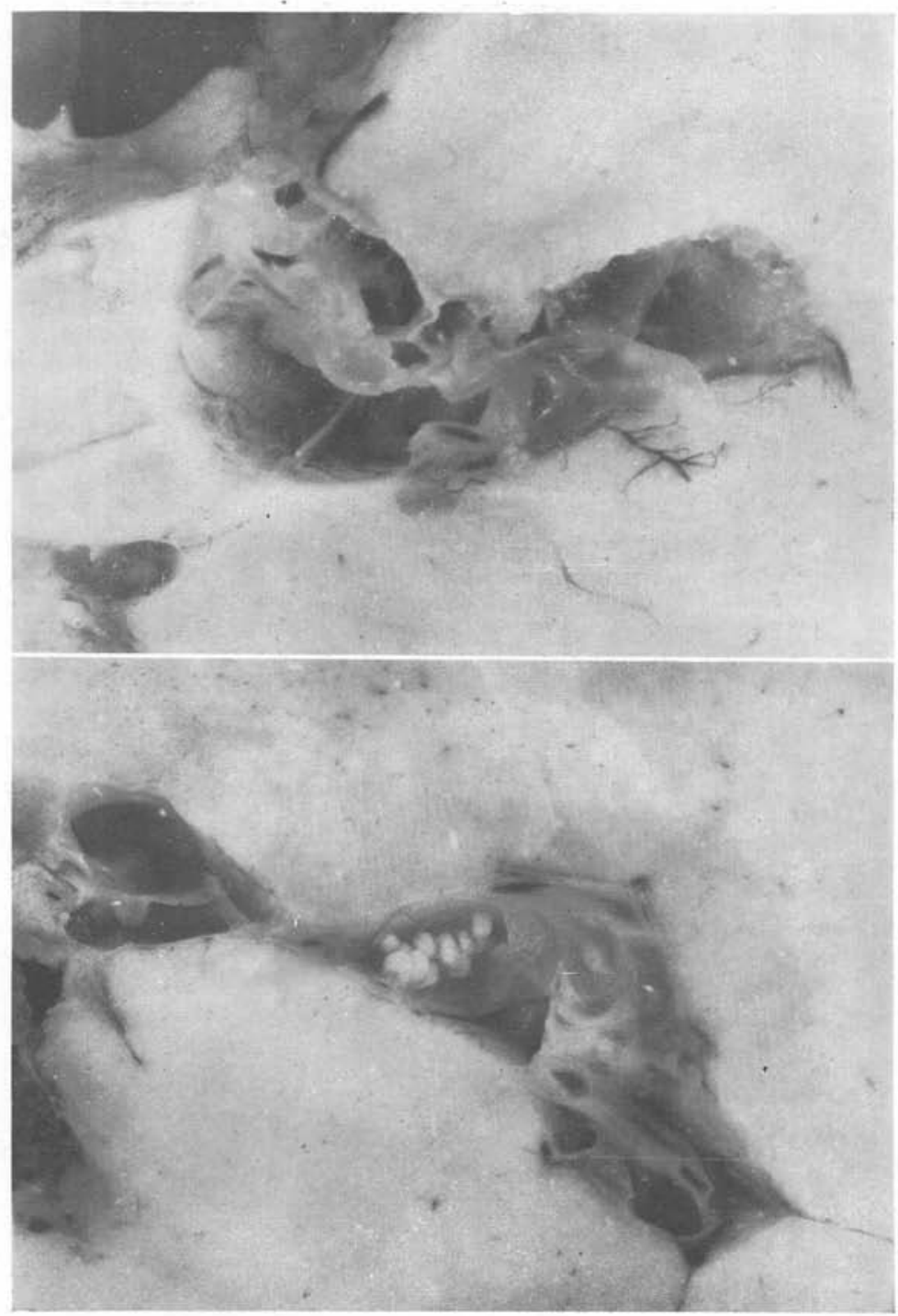

Fig. 13 (En haut), - Cénure de la scissure sylvienne.

Fig. 14 (En bas). - Coupe passant par le pulvinar et sillon de l'hippocampe. 


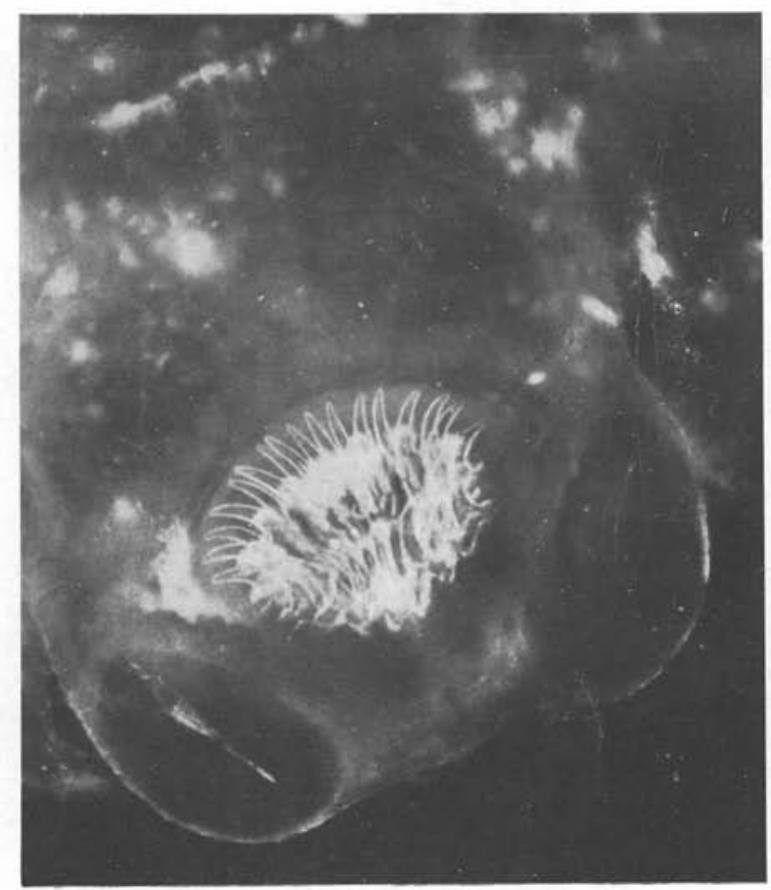

Fic. 15. - Scolex d'un cénure normal.

chets, au nombre iotal de 28 en moyenne (fig. 18). Les grands crochets (fig. 19) ont $142 \mu$ de longueur totale moyenne. La lame est peu courbée. Les bords du manche sont presque parallèles avec un très léger renflement à la base. Le bord dorsal est parfaitement régulier, dépourvu de sinuosités. Les petits crochets (fig. 19) mesurent en moyenne $108 \mu$ de longueur totale ; la lame est nettement plus courbée que celle des grands crochets.

Pour nous mettre dans des conditions analogues à celles de Baylis (1932), qui a également étudié un cas analogue de cénurose, nous avons appliqué la formule de Meggitt (fig. 20, tableau 1). L'analyse des chiffres de Baylis et nos propres constatations nous obligent à reconnaître l'impossibilité d'utiliser cette méthode efficacement pour la diagnose des crochets de cénure.

Comme nous l'avons dit ci-dessus, la proportion des vésicules normales est très faible par rapport à celles qui ne présentent pas de scolex ou en montrent de simples ébauches plus ou moins formées (fig. 21). Remarquons que, même dans les cénures récoltés chez les 

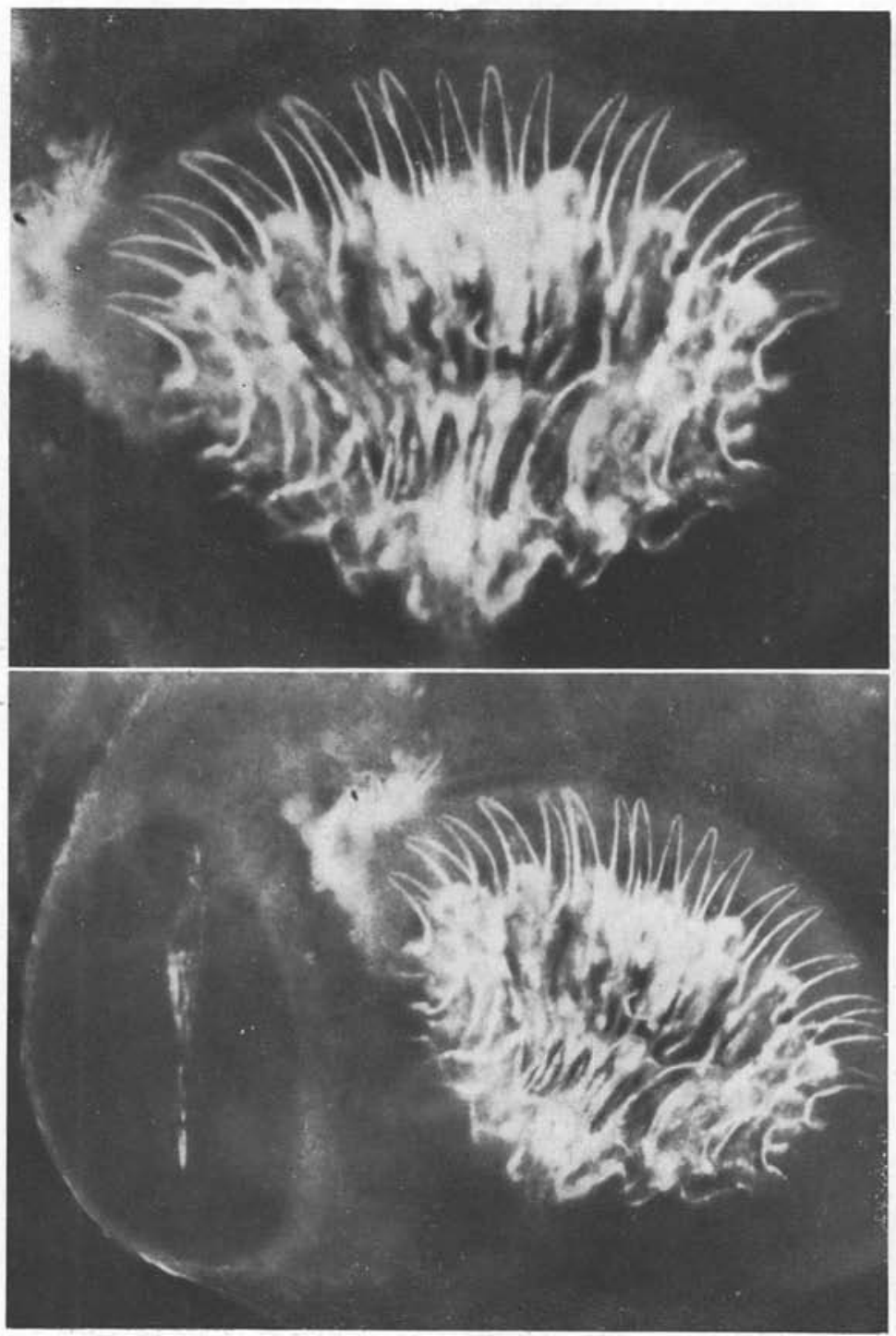

Fig. 16 (En haut) et 17 (En bas). - Même scolex que fig. 15 , à plus fort grossissement. 


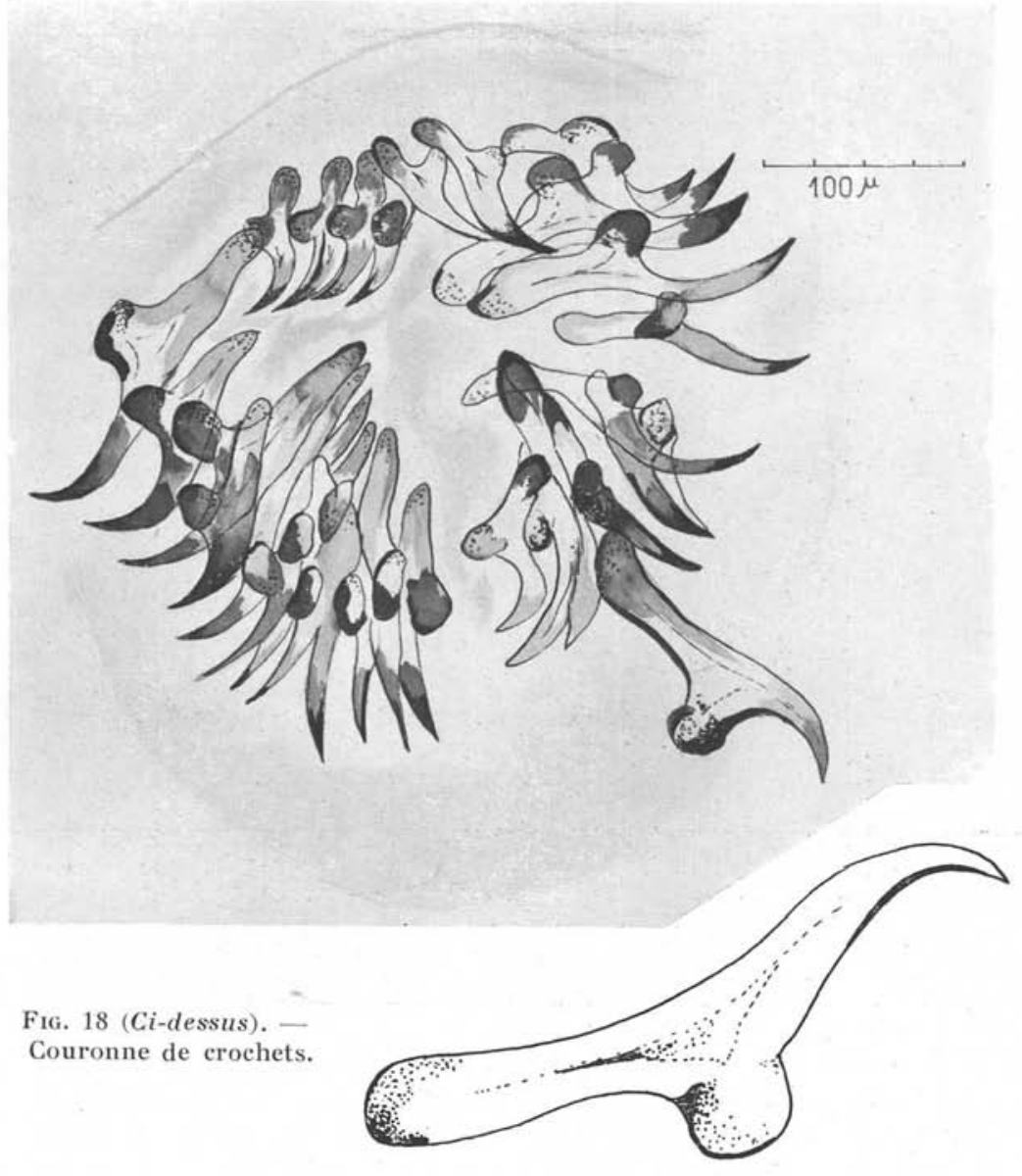

FIG. 18 (Ci-dessus). -

Couronne de crochets.

$50 \mu$

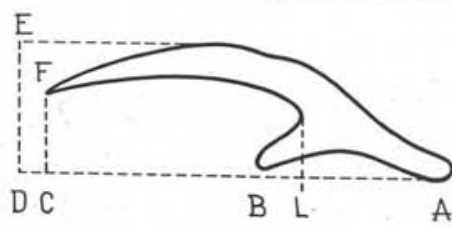

FIG. 20. - Schéma, d'après Meggitt (in Baylis) pour établissement de sa formule.

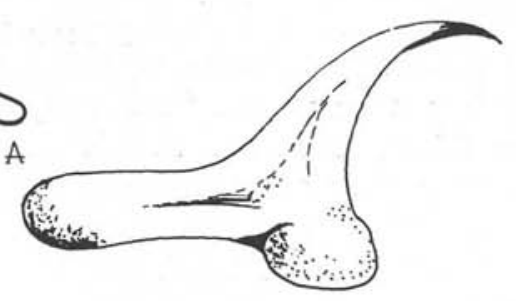

Fig. 19 (Ci-contre). - Grand et petit crochets isolés. 


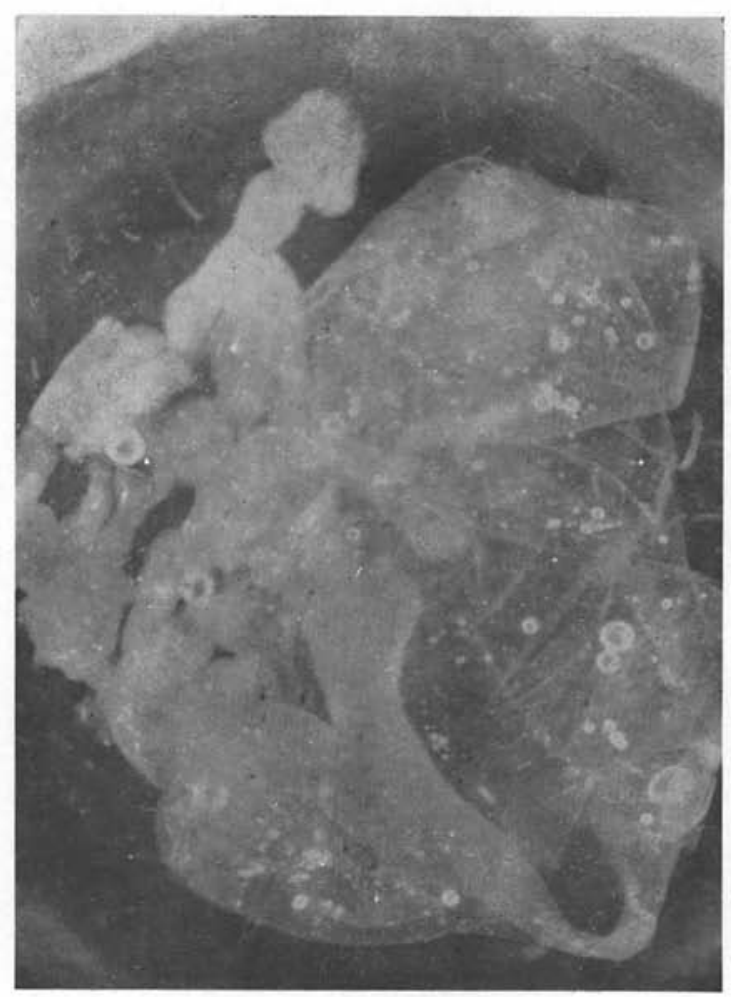

Fig. 21. - Cénure dégénéré.

hòtes normaux, il n'est pas rare de constater la présence d'un certain nombre de scolex mal conformés, inaptes à évoluer ultérieurement chez le carnivore.

\section{Discussion}

Le diagnostic de la cénurose repose sur l'examen morphologique du parasite et sur sa localisation, à laquelle est liée l'histoire de la maladie qu'il occasionne.

Nous ne retenons ici, comme cénures de l'homme, que deux espèces convenablement identifiées: Cœnurus multiceps Goeze (syn. Conurus cerebralis Rud.) et Cœnurus serialis Gervais. Le premier est la larve de Tænia multiceps Leske 1780 , et le second celle de Tænia serialis Gervais 1847. 
Tableau I

\begin{tabular}{|c|c|c|c|c|c|c|c|c|c|}
\hline & & \multicolumn{4}{|c|}{ GRANDS CROCHETS } & \multicolumn{4}{|c|}{ Petits сиоснетs } \\
\hline & & $A L$ & AC: & $\mathrm{FC}$ & EI) & $\mathrm{AL}$ & $\mathrm{AC}$ & $\mathrm{FC}$ & ED \\
\hline \multirow{3}{*}{\multicolumn{2}{|c|}{$\begin{array}{l}\text { Taramelli } \\
\text { Dubois }\end{array}$}} & 087 & 156 & 051 & 064 & 085 & 190 & 056 & 082 \\
\hline & & 092 & 167 & 054 & 073 & 087 & $15 \mathrm{x}$ & 058 & 074 \\
\hline & & 090 & 168 & 058 & 073 & 0.6 & 168 & 043 & 071 \\
\hline \multirow{3}{*}{$\begin{array}{l}T . \text { serialis } \\
\text { lapin }\end{array}$} & l & 090 & 147 & 055 & 072 & $0: 8$ & 131 & 051 & 078 \\
\hline & & 093 & 150 & 055 & $07 \mathrm{w}$ & 077 & 113 & 061 & 082 \\
\hline & 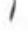 & 093 & $14 \mathrm{~s}$ & 055 & 068 & 083 & 150 & 067 & 083 \\
\hline \multirow{3}{*}{$\begin{array}{l}\text { T. multiceps } \\
\text { mouton }\end{array}$} & 1 & 093 & 18.5 & 045 & 070 & 086 & 161 & 052 & 079 \\
\hline & & 095 & 190 & 043 & 070 & 079 & 153 & 067 & 086 \\
\hline & & 093 & $1 \times 1$ & 041 & 066 & 083 & 153 & 057 & 079 \\
\hline \multirow{3}{*}{\multicolumn{2}{|c|}{$\begin{array}{l}T . \text { mult. } \\
\text { Crochets } \\
\text { incomplèt. } \\
\text { formès } \\
\text { mouton }\end{array}$}} & & & & & & & & \\
\hline & & 096 & 177 & 077 & 087 & 1184 & 181 & 103 & 122 \\
\hline & & 093 & 193 & 076 & 089 & 085 & 176 & 100 & 112 \\
\hline \multirow{3}{*}{\multicolumn{2}{|c|}{ Cas personnel. }} & 085 & 130 & 045 & 055 & 055 & 100 & 045 & 055 \\
\hline & & 091 & 149 & 052 & 062 & 049 & 104 & 048 & 060 \\
\hline & & 094 & 156 & 058 & 068 & 052 & 1114 & 019 & 061 \\
\hline Moyennes & & 090 & 142 & 051 & 061 & 052 & 103 & 047 & 0.2 \\
\hline
\end{tabular}

Turner et Leiper (1919), Taramelli et Dubois (1932) ont assimilé ces cénures humains à C. glomeratus Railliet et Henry, 1915, en se basant sur la description originale des crochets donnée par ces auteurs. Mais, ultérieurement, Railliet et Marullaz (1919) ont rectifié leurs données primitives, faussées par une erreur matérielle de mensuration. Il s'ensuit que les nouvelles dimensions ne cadrent plus avec celles des cénures de Turner et Leiper, ainsi que de Taramelli et Dubois. Baylis (1932) a revu les pièces de Taramelli et Dubois et n'a pu affirmer la diagnose, envisageant même la possibilité d'une nouvelle espèce. D'ailleurs, d'après Crusz (1947), la cénurose humaine serait occasionnée uniquement par C. multiceps ; C. serialis ne représentant qu'une souche physiologique spéciale et $C$. glomeratus une forme insuffisamment développée. Nous pensons que les localisations différentes de $C$. multiceps et $C$. serialis sont suffisantes pour les séparer, car elles ont une analogie frappante avec celles observées chez leurs hôtes normaux (mouton et lapin), correspondant à deux vers adultes distincts. Il est plus difficile de se pronon- 
cer sur la valeur taxinomique de $C$. glomeratus, dont l'adulte n'est pas décrit, à notre connaissance.

Caractères morphologiques. - Les crochets de T. multiceps et de $T$. serialis sont très ressemblants entre eux. On admet classiquement qu'ils se distinguent par leur longueur : 150 à $170 \mu$ et 90 à $130 \mu$ pour ceux de $T$. multiceps; 130 à $157 \mu$ et 85 à $112 \mu$ pour ceux de $T$. serialis. Comme on le voit, ces dimensions chevauchent l'une sur l'autre. Nous avons vu ci-dessus que les tableaux fournis par les formules de Meggitt ne donnent pas de renseignements suffisants pour distinguer les deux espèces. La forme présente quelques différences : le manche des grands crochets est droit chez $T$. multiceps, sinueux chez T. serialis. Mais ce caractère se voit mieux chez les vers adultes, où les crochets sont complètement développés, que chez leurs larves. Rappelons que d'autres caractères distinctifs existent aussi dans les formes sexuées, notamment en ce qui concerne la disposition des branches utérines des anneaux mûrs.

En somme, un examen morphologique des crochets ne constitue le plus souvent qu'un caractère différentiel imparfait. C'est ainsi que, dans notre cas, il est impossible d'utiliser leurs dimensions qui peuvent être rapportées aussi bien à $T$. multiceps qu'à $T$. serialis. Cependant, la forme du manche et la courbure de la lame sont nettement en faveur de T. multiceps.

Pour que la diagnose fût à l'abri de toute critique, il faudrait. partant du cénure humain, infester expérimentalement un chien neuf, et, après obtention d'anneaux mûrs du ver adulte chez cet animal, conférer la cénurose à un hôte intermédiaire normal, également neuf. On devrait retrouver un cénure typique dans sa localisation habituelle. Malheureusement, il est difficile de réunir ces conditions idéales, ce qui exigerait la présence continuelle d'animaux neufs au laboratoire, la cénurose humaine, maladie considérée comme très rare jusqu'à présent, étant toujours observée inopinément. Bonnal, Joyeux et Bosch (1933) ont infesté un jeune chien avec un cénure humain à $T$. serialis ; mais, n'étant pas absolument certains que ce carnivore était indemne, ils ont été obligés de le sacrifier au bout de 12 jours de surveillance après ingestion et ont pu observer de jeunes ténias dont la longueur correspondait à un début de développement. Leur expérience prouve la vitalité du cénure chez un hòte anormal.

Localisation du parasite. - Cette localisation représente un excellent caractère pour distinguer les deux cénures. $C$. multiceps s'observe dans l'encéphale et quelquefois dans la moelle épinière ; 
C. serialis se localise dans le tissu conjonctif, où il est souvent superficiel, ainsi que dans les grandes séreuses. De ce tropisme électif, découle une histoire médicale différente pour chacun des deux parasites.

C. multiceps provoque de graves accidents nerveux pouvant entraîner la mort. Chez son hôte normal, le mouton, il occasionne le tournis, trop connu pour être décrit ici ; son étude se trouve dans les ouvrages classiques.

Chez l'homme, il s'agit également de déterminations névraxitiques graves, fréquemment mortelles. Le plus souvent, c'est au niveau de l'encéphale que siège le cénure. Il a une tendance, partant de la région basilaire, à s'infiltrer dans les scissures, plus particulièrement dans les vallées sylviennes, d'où troubles de compression cérébrale extrêmement sérieux, s'accompagnant habituellement de dilatation des ventricules. L'envahissement parasitaire parait se faire essentiellemnt en suivant la leptoméninge. Pierre Marie, Foix et Brumpt (1913), Culver (1941), Clapham (1941) ont noté des localisations intra-ventriculaires.

Buckley (1947) a extirpé un cénure de la moelle épinière chez une fillette de 14 ans ayant séjourné dans une région d'élevage de moutons (Pays de Galles). Toutefois, la diagnose du parasite n'a pu être établie de façon précise par $\mathrm{H}$. Crusz.

D'une façon générale, le pronostic de la cénurose nerveuse demeure très sombre. Cependant, les récents progrès de la neuro-chirurgie permettront peut-être d'obtenir des résultats thérapeutiques plus favorables (voir les trois guérisons des malades de Becker et Jacobson) et, en même temps, de déceler plus fréquemment cette affection parasitaire.

C. serialis, au contraire, ne provoque généralement que des accidents bénins. Parfois, chez son hòte normal (léporidé), il peut, lorsqu'il atteint un volume considérable, causer des accidents de compression. En pathologie humaine, il s'agit le plus souvent de tumeurs superficielles, pouvant entrainer une simple gêne fonctionnelle et s'extirpant chirurgicalement avec facilité. Dans une seule observation de Brumpt, Duvoir et Sainton (1934), reproduite par divers auteurs classiques, il a été noté une cachexie progressive, avec cyanose, subictère, incontinence des sphincters et finalement anurie ayant entrainé la mort. A l'autopsie, foyer d'hépatisation de la base du poumon gauche, atrophie considérable du foie à surface granuleuse et irrégulière. En même temps, présence de trois nodules dans les régions suivantes : sous-scapulaire droite, cuisse gauche, juxta-iliaque droite, le volume variant d'une noix à une mandarine. 


\section{TABLEAU II}

\begin{tabular}{|c|c|c|c|c|}
\hline AUTEURS & PAYS & $\begin{array}{l}\text { ANTÉCÉDENTS } \\
\text { DU MALADE }\end{array}$ & $\begin{array}{l}\text { Localisation } \\
\text { DU Parasite }\end{array}$ & IDENTIFICATION \\
\hline $\begin{array}{l}\text { P. Marie, Foix, } \\
\text { Brumpt }(1913) \ldots\end{array}$ & France & $\begin{array}{l}\text { Homme, } \\
\text { serrurier }\end{array}$ & Ventricule latéral & C. multiceps \\
\hline $\begin{array}{c}\text { Turner, } \quad \text { Leiper } \\
\left({ }^{9} 919\right) \ldots \ldots \ldots \ldots \ldots\end{array}$ & $\begin{array}{c}\text { Nord } \\
\text { Nigeria }\end{array}$ & & $\begin{array}{c}\text { Muscles } \\
\text { intercostaux }\end{array}$ & C. glomeratus \\
\hline $\begin{array}{r}\text { Taramelli, Dubois. } \\
(1919) \ldots \ldots \ldots \ldots \ldots\end{array}$ & Congo belge & Femme & $\begin{array}{l}\text { Tissu sous-cutané, } \\
\text { avant-bras }\end{array}$ & C. glomeratus \\
\hline $\begin{array}{c}\text { Bonnal, Joyeux, } \\
\text { Boseh (1933)..... }\end{array}$ & Marseille & $\begin{array}{l}\text { Femme, } \\
\text { promiscuite } \\
\text { chiens }\end{array}$ & Fesse droite & C. serialis \\
\hline $\begin{array}{l}\text { Brumpt, Duvoir, } \\
\text { Sainton (1934)... }\end{array}$ & France & $\begin{array}{l}\text { Femme, } \\
\text { promissuité } \\
\text { chiens }\end{array}$ & $\begin{array}{l}\text { Trois localisations } \\
\text { superficielles }\end{array}$ & C. serialis \\
\hline Culver (1941) ...... & Afrique Sud & Femme & $\begin{array}{l}\text { Ventricule cèr. } \\
\text { gauche }\end{array}$ & C. multiceps \\
\hline Clapham (1941) ... & Angleterre & $\begin{array}{l}\text { Homme région } \\
\text { méditerran. }\end{array}$ & $\begin{array}{l}\text { Corne postérieure } \\
\text { ventricule latéral }\end{array}$ & c. multiceps \\
\hline Cannon $(1942 ; \ldots$. & Nord Nigeria & $\begin{array}{l}\text { Homme } \\
\text { chasseur }\end{array}$ & $\begin{array}{l}\text { Triceps } \\
\text { bras droit }\end{array}$ & $?$ \\
\hline $\begin{array}{c}\text { Roger, } \\
\text { Sautet, } \\
\text { Paillas (1942)... }\end{array}$ & Marseille & Femme & $\begin{array}{c}\text { Fosse cérébrale } \\
\text { postérieure }\end{array}$ & C. multiceps (?) \\
\hline $\begin{array}{c}\text { Buckley, } \quad \text { Crusz } \\
(1947) \ldots \ldots \ldots \ldots\end{array}$ & Angleterre & $\begin{array}{l}\text { Fille } 14 \text { ans. } \\
\text { Séjour } \\
\text { Pays de Galles }\end{array}$ & Moelle épinière & ? \\
\hline $\begin{array}{r}\text { Harant, Midrouil- } \\
\text { let (1949) } \ldots \ldots \ldots \ldots\end{array}$ & France (Tarn) & $\begin{array}{l}\text { Homme, } \\
\text { région élevage }\end{array}$ & Deltoïde & C. serialis \\
\hline $\begin{array}{r}\text { Johnstone, Jones } \\
(1950) \ldots \ldots \ldots \ldots \ldots\end{array}$ & U.S.A. & $\begin{array}{c}\text { Enfant mâle } \\
26 \text { mois }\end{array}$ & $\begin{array}{c}\text { Cérébrale } \\
\text { pont pédoncules }\end{array}$ & C. multiceps \\
\hline $\begin{array}{r}\text { Becker, Jecobson } \\
(1950) \ldots \ldots \ldots \ldots \ldots\end{array}$ & Afrique Sud & $\begin{array}{l}\text { Homme, } \\
\text { région élevage }\end{array}$ & Cétébrale & C. multiceps \\
\hline id. & id. & id. & id. & id. \\
\hline id. & id. & id. & id. & id. \\
\hline $\begin{array}{l}\text { Ranque, Nicoli, } \\
\text { Duplay (présen- } \\
\text { te observation). }\end{array}$ & $\begin{array}{l}\text { Alpes- } \\
\text { Maritimes }\end{array}$ & $\begin{array}{l}\text { Hom me } \\
\text { berger }\end{array}$ & Cérebrale & c. multiceps \\
\hline
\end{tabular}


Il s'agissait de $C$. serialis. Nous pensons que les cénures en question, à localisation essentiellement superficielle, étaient des éléments sans rapport avec l'affection viscérale qui a emporté la malade.

Nous complétons ci-dessous le tableau (tableau 2) de Johnstone (1950) en mentionnant les nouvelles observations de cénurose humaine parues depuis la publication de son travail. Nous citons les deux cas de $C$. glomeratus avec les réserves indiquées plus haut.

\section{RÉSUMÉ}

Nous relatons la neuvième observation de cénurose cérébrale humaine. Dans les cas publiés jusqu'à présent, l'identification exacte de l'agent causal n'a pas toujours pu être précisée. Nous rapportons le nôtre à $C$. multiceps, larve de $T$. multiceps Leske, 1780 , en nous basant principalement sur la forme des crochets et sur la localisation du cénure.

Comme l'homme est un hôte anormal de cette larve, les scolex présentent le plus souvent une morphologie aberrante; il a été nécessaire d'en examiner un très grand nombre, pour en trouver qui soient identifiables.

\section{Travaux cITÉs}

Baylis (H.-A.). - On a Cænurus from Man. Trans. R. Soc. trop. Med. and Hyg., 25, 1932, p. 275-280.

Becker (B. J. P.) and Jacobson (S.). - Infestation of the Human Brain with Conurus cerebralis. Lancet, h, 1951, p. 198-202.

Bonnal (G.), Joyeux (Ch.) et Bosch (P.), - Un cas de cénurose à Multiceps serialis. Bull. Soc. Path. exot., 26, 1933, $\mathrm{n}^{\circ}$ 8, p. 1060-1071.

Brumpt (E.). - Précis de Parasitologie, 5éd., p. 738-745.

Brumpt (E.), Duvoir (M.-E.) et Sainton (J.). - Un cas de cénurose humaine due à Conurus serialis, parasite habituel des lapins et des lièvres. Bull. Acad. Méd., 12, 10 juillet 1934, n² 26, p. 56-67.

Buckley (J. J. C.). - Canurus from human spinal cord. Trans. R. Soc. trop. Med. and Hyg., $1947, \mathrm{n}^{\circ} 1$, p. 7.

Cannon (D. A. B.). - A case of human Infection with a species of Conurus. Ann. trop. Med. and Parasit., 255, 1942, $\mathrm{n}^{\circ} 2$, p. 747-750.

Clapham (P. A.). - An english Case of Conurus cerebralis in the Human Brain. Jrn. Helminthol., 19, december 1941, 3/4, p. 84-86.

Crusz (H.). - On a english Case of anintramedullary spinal Cœnurus in Man with some remarks on the identity of Canurus sp. infesting Man. Jrn. Helminthol., 22, 1948, $\mathrm{n}^{\circ} 2$, p. 73-76.

Johnstone (H. G.) and Jones (O. W. Jr). - Cerebral cenurosis in an infant. Amer. Jrn. trop. Med., 30, 1950, n 3, p. 431-441. 
Harant (H.) et Midrouillet (M.). - Sur un nouveau cas de cénurose humaine. Bull. Soc. Path. exot., 42, 1949, n 5-6, p. 173-174.

Joyeux (Ch.) et Baer (J. G.). - Faune de France, 30, 1936. Cestodes. Lechevalier éd.

Neveu-Lemaire (M.). - Traité d'Helminthologie médicale et vétérinaire. Vigot éd., Paris, 1936.

Railliet (A.). - Traité de Zoologie médicale et agricole. $2^{\circ}$ éd., 1895.

Railliet (A.) et Marullaz (M.). - Sur un cénure nouveau du Bonnet chinois (Macacus sinicus). Bull. Soc. Path. exot., 12, 1919. p. 223-228.

Roger (H.), Sautet (J.) et Paillas (J.-E.). - Un cas de cénurose de la fosse cérébrale postérieure. Rev. neurol., 7/, 1942, p. 319-321.

Taramelli (R.) et Dubois (A.). - Un cas de cénurose chez l'Homme. Ann. Soc. belge de Méd. trop., 11, 1931, $\mathrm{n}^{\circ} 2$, p. 151-154.

Turner (M.) et Leiper (R. T.). - On the occurence of Canurus glomeratus in Man West Africa. Trans. R. Soc. trop. Med. and Hyg., 13, 1919, p. 23-24.

(Faculté de Médecine, Laboratoire de Parasitologie, Marseille) 